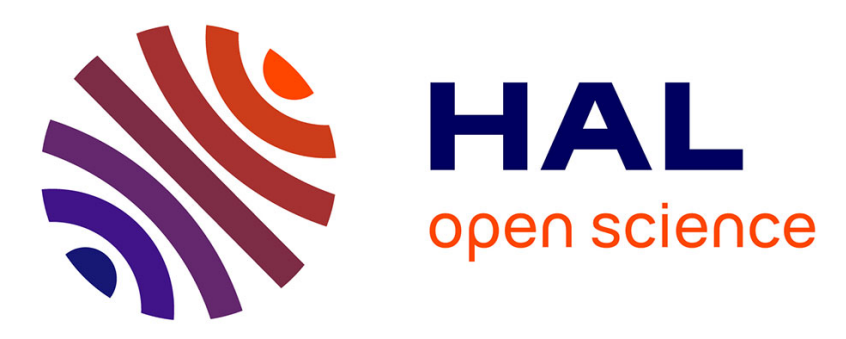

\title{
Language and Variety Verification on Broadcast News for Portuguese
}

\author{
Jean-Luc Rouas, Isabel Trancoso, Céu Viana, Mónica Abreu
}

\section{To cite this version:}

Jean-Luc Rouas, Isabel Trancoso, Céu Viana, Mónica Abreu. Language and Variety Verification on Broadcast News for Portuguese. Speech Communication, 2008, 50 (11-12), pp.965. 10.1016/j.specom.2008.05.006 . hal-00499218

\section{HAL Id: hal-00499218 https://hal.science/hal-00499218}

Submitted on 9 Jul 2010

HAL is a multi-disciplinary open access archive for the deposit and dissemination of scientific research documents, whether they are published or not. The documents may come from teaching and research institutions in France or abroad, or from public or private research centers.
L'archive ouverte pluridisciplinaire HAL, est destinée au dépôt et à la diffusion de documents scientifiques de niveau recherche, publiés ou non, émanant des établissements d'enseignement et de recherche français ou étrangers, des laboratoires publics ou privés. 


\section{Accepted Manuscript}

Language and Variety Verification on Broadcast News for Portuguese

Jean-Luc Rouas, Isabel Trancoso, Céu Viana, Mónica Abreu

PII:

S0167-6393(08)00075-7

DOI:

10.1016/j.specom.2008.05.006

Reference:

SPECOM 1717

To appear in:

Speech Communication

Received Date:

14 June 2007

Revised Date:

8 May 2008

Accepted Date:

13 May 2008

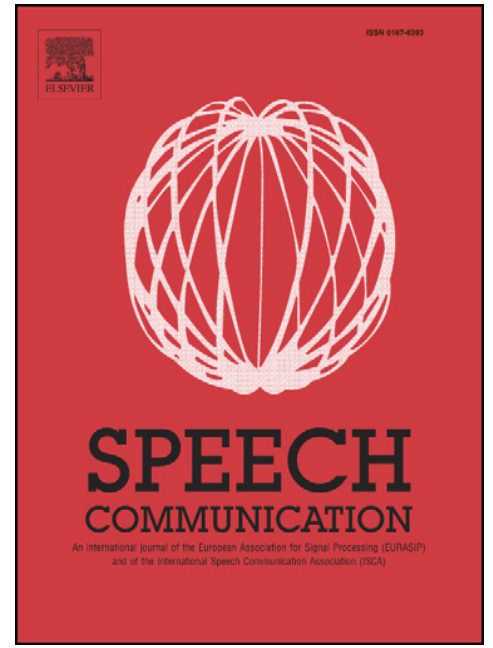

Accepted Date:

Please cite this article as: Rouas, J-L., Trancoso, I., Viana, C., Abreu, M., Language and Variety Verification on Broadcast News for Portuguese, Speech Communication (2008), doi: 10.1016/j.specom.2008.05.006

This is a PDF file of an unedited manuscript that has been accepted for publication. As a service to our customers we are providing this early version of the manuscript. The manuscript will undergo copyediting, typesetting, and review of the resulting proof before it is published in its final form. Please note that during the production process errors may be discovered which could affect the content, and all legal disclaimers that apply to the journal pertain. 


\title{
Language and Variety Verification on Broadcast News for Portuguese
}

\author{
Jean-Luc Rouas ${ }^{1,2}$, Isabel Trancoso ${ }^{1}$, Céu Viana ${ }^{3}$, Mónica Abreu ${ }^{1}$ \\ ${ }^{1}$ INESC-ID, Spoken Language Systems Laboratory (L2F), R. Alves Redol , 9, 1000-029 \\ Lisboa, Portugal \\ ${ }^{2}$ INRETS Electronic, Waves and Signal Processing Research Laboratory for Transport, \\ 20 Rue Élisée Reclus, 59650 Villeneuve d'Ascq, France \\ ${ }^{3}$ Centro de Linguística da Universidade de Lisboa, Av. Prof. Gama Pinto, 2, 1649-003 \\ Lisboa - Portugal
}

\begin{abstract}
This paper describes a language/accent verification system for Portuguese, that explores different type of properties: acoustic, phonotactic and prosodic. The two-stage system is designed to be used as a pre-processing module for the Portuguese Automatic Speech Recognition (ASR) system developed at INESC-ID. As the ASR system is applied everyday to transcribe the evening news from a Portuguese public TV channel, the presence of other languages (mainly English) and other varieties of Portuguese is very likely. In the first stage, for each automatically detected speaker, the system verifies if the spoken language is Portuguese, as opposed to nine other languages - English, Belgian Dutch, Croatian, Czech, Galician, Greek, Hungarian, Sloven and Slovak. The identified Portuguese speakers are then fed to the second stage which aims at identifying the Portuguese variety: European, Brazilian or African Portuguese from 5 countries. The identification results are then used either to mark the speech data as untranscribable or forward it to the European Portuguese ASR system, or a system tuned for other languages or varieties. The language verification system achieved an equal error rate for European Portuguese of $2.5 \%$. In terms of variety identification, the overall rate of correct identification was $83.9 \%$, when considering only the 3 broad varieties, and the best results were obtained for Brazilian Portuguese, also the variety that proved easiest to identify in perceptual experiments. The identification rate between African varieties themselves is relatively low, a fact that was also observed in the perceptual experiments.
\end{abstract}

Key words: Language verification, Portuguese varieties.

Email address: rouas@inrets.fr, Isabel.Trancoso@inesc-id.pt, mcv@clul.ul.pt, monica7abreu@gmail.com (Jean-Luc Rouas ${ }^{1,2}$, Isabel Trancoso $^{1}$, Céu Viana ${ }^{3}$, Mónica Abreu ${ }^{1}$ ). 
The Spoken Language Systems Lab (L2F) of INESC-ID has been actively working on Automatic Speech Recognition (ASR) for many years. The Portuguese ASR system is currently applied to the transcription of the broadcast news extracted from a public national channel, the "Telejornal" on RTP1. The system is working on a daily basis and results of the transcription of the last broadcasted evening news are available at http://www.l2f.inesc-id.pt/wiki/index.php/Demos.

However, one of the problems encountered by the ASR system is the presence of different languages: many interviews are subtitled in Portuguese, while the audio remains in the original language. This generates a long stream of errors which can have a very negative impact on any modules that follow the recognition module (search, indexation, summarization, etc.). Therefore the system needs to know if the spoken language is really Portuguese or another language. Furthermore, if several ASR systems are available for the most frequent other languages (like English), this also allows the selection of the most appropriate ASR system. Moreover, in case the Portuguese language is identified, we also have to determine which variety of Portuguese is actually spoken, as there may be great variations in pronunciation.

This paper is organized as follows: We start by recalling the cues commonly used for language and accent characterization (section 1). Then we make a short review of state-of-the-art systems for language and accent identification (section 2). Section 3 is dedicated to a discussion about the differences between Portuguese varieties. The design of the language verification system is detailed in section 4 . The corpora used for the experiments on language and variety identification are described in section 5. Experiments on language verification are discussed in section 6 , focusing mainly on the performances of the Portuguese language verifier. In section 7 , we study how the language verification system reacts when provided with samples of different varieties of Portuguese. Finally, the performance of the variety verification system is discussed in section 8 .

\section{Introduction}

The aim of automatic language identification (LID) is to find which language is spoken in an utterance pronounced by an unknown speaker. Several cues can be used for this purpose, based on linguistic and perceptual studies on the differences among languages.

In [47], four kinds of cues are described:

- Phonology: The phoneme sets used in different languages differ, even though many languages share a common subset. Phonotactics, i.e. the rules governing the sequences of phonemes are also different.

- Morphology: The words roots and lexicons differ from one language to another. 
Each language has its own vocabulary.

- Syntax: The way to construct sentences is different among languages.

- Prosody: Rhythm and intonation patterns are different.

Phonological properties are used in the most common language identification systems:

- The Acoustic Language Identification Systems use the differences in acoustic realizations of phonemes.

- The PRLM (Phone recognition followed by language modeling) Systems or PPRLM (Parallel-PRLM) Systems characterize each language by its most frequent sequences of phones.

Morphological and syntactical cues are hard to deal with if we do not have the transcription output of a speech recognition system, which is a language-specific task.

Prosody is also hard to model, mostly because of the suprasegmental nature of the prosodic features. That is why prosody has seldom been used for high performance language identification. More recently, however, prosodic features are beginning to be integrated in many systems, conjointly with acoustic or phonotactics, in order to take into account all the available information [44].

\section{Actual performances for Language verification systems}

Despite the growing interest on language identification that was observed during the 1980s, this area has not been much considered in the following decade. Nowadays, there is a regain of interest for language identification systems, probably motivated by their potential application in surveillance. This interest also led to significant improvement in performances as shown by the more recent NIST evaluations. The task addressed by these evaluations are however different from "classic" language identification experiments.

Traditionally, the language identification systems were asked to identify a language within a finite set of languages. Since the 1996 NIST Language Recognition Evaluation, the task has moved to language verification, which is similar to speaker verification: the aim is to evaluate if the speech excerpts belong to a target language, or not.

As the NIST evaluations are a very good ground for estimating language verification system performances, some of the best performing systems of the 2005 evaluation are briefly described below (see www. nist.gov/speech/tests/lang/for a complete list of participating organizations). 
The 7 languages used in the NIST 2005 Evaluation were the following: English (American \& Indian), Hindi, Japanese, Korean, Mandarin (Mainland \& Taiwan), Spanish (Mexican) and Tamil. The evaluation of the system is achieved using Equal Error Rate (or EER), which means balanced errors between false alarms and missed detections. Usually, a detection error trade-off curve (or DET-curve) is also provided as a characteristic of the performances of the tested system. Of the several language verification systems used in the NIST 2005 Evaluation campaign, the best performing ones use either acoustic or phonotactic (P-PRLM) approaches or a fusion of both.

For example, the Brno university system (described in [24]) uses a GMM-based acoustic system, with discriminatevely trained models, combined with a Neural Network-based PPRLM system. Combining the scores of these approaches with a weighted addition of the log-likelihoods gives an overall equal error rate of $5.0 \%$ on 30-second excerpts.

The system submitted by the Georgia Institute of Technology and the Infocomm Institute uses a fusion of two approaches [22]. The first one is a classical PPRLM. The second approach uses a "Bag of Sound" (BOS) recognizer, which can be also called "universal phone recognizer". This BOS recognizer is trained to recognize 258 phonemes from 6 languages (English, Mandarin, Japanese, Hindi, Spanish and German). Then, SVM classifiers are used to make pairwise decisions. The scores obtained from both approaches are concatenated to form a score vector which is fed to the back-end system. Two approaches are used to provide scores for each of the target languages: Artificial Neural Networks or Linear Discriminant Functions. The results obtained by each of these classifiers are then merged. The performance obtained with this system is 12\% EER on the NIST 200530 -second excerpts.

The Lincoln Laboratory of the Massachusetts Institute of Technology has presented a fusion of several systems [6]. The systems were: GMM-SDC (Gaussian Mixture Models with Shifted Delta Cepstra Features), SVM-SDC (Support Vector Machine with Shifted Delta Cepstra Features), PPRLM (Parallel Phone Recognition followed by n-gram Language Models classifiers), PPRLM-lattice (Parallel Phone Recognition followed by n-gram Language Models classifiers using Phone Lattices [15]), PPRSVM-lattice (Parallel Phone Recognition followed by Support Vector Machine classifiers using Phone Lattices), and PPRBT (Parallel Phone Recognition followed by Binary Tree Language Models (developed at IBM)). The fusion is achieved by modeling the concatenated output scores of each of these systems by Gaussian Mixture Models. The performances reached by this system is $4.2 \%$ of Equal Error Rate on 30 seconds test utterances.

All these systems show the performance that is achieved nowadays on the language verification task. While being the best performing systems, PPRLM are also the most complex ones (both in terms of design and computational time). In fact, building a powerful PPRLM system almost requires the implementation of speech recog- 
nizers for several languages. The acoustic modeling systems have been thoroughly investigated during last years, taking benefits from speaker verification researches, and are now almost competitive with PPRLM systems. It is however noticeable that none of these systems use prosodic features.

Dialect identification is a somewhat harder topic than language identification and has not been for the moment as much investigated [23] [4] [14] [36] [43] [45] [17] [16], although one can find a growing number of references on a related problem - foreign accent identification [42]. Many approaches use language identification systems applied to native dialect identification.

For example, in [39] a GMM-SDC based system is applied to Spanish dialects identification, considering only two dialects (Cuban and Peruvian) with the "Miami" corpus. On this data, the system generates an error rate over 30\%. This experiment has also been carried on the dialects present in the CallFriend corpus, using 30 seconds utterances: American English (North vs. South), Chinese (Mandarin vs. Taiwan) and Spanish (Caribbean vs. Non-Caribbean). The error rate were respectively: $15.0 \%$ for American English, 11.5\% for Chinese and $13.7 \%$ for Spanish.

In [8], another GMM-based system is applied to Chinese dialect identification. The accents present is this corpus come from 4 regions: Beijing, Shanghai, Guangdong and Taiwan. The data used come from the "Multi accent Mandarin corpus", consisting in 1440 speakers for approximately 16 hours. 60 speakers were used for testing for each dialect. The results were between $12 \%$ and $15 \%$ errors (for female and male speakers respectively) for utterances of approximately 20 seconds.

The experiments reported in [40] concern also the identification of Chinese dialects. Here the considered dialects are Mandarin, Holo and Hakka (all spoken in Taiwan). The corpus used in these experiments is quite small, with a total of 8 speakers reading 30 paragraphs, generating sentences about 15 seconds long. The same speakers are used for training and testing, and each speaker read each text 3 times, once in each of the dialects. Using MFCC and pitch features and a Gaussian mixture bigram model, the system achieves a performance of $94 \%$ of correct identifications. These experiments however show the importance of considering prosodic information, as using only the pitch-based features, the identification rate is $57 \%$.

The prosodic system developed in the $\mathrm{PhD}$ thesis of the first author [31] was successfully tested on read speech from 7 languages (English, French, German , Italian, Spanish from the original Multext corpus [7], Mandarin Chinese [19] and Japanese [18]), achieving around $70 \%$ of correct identification [32]. The system was also tested on semi-spontaneous Arabic dialects (Araber database [34]), where the the task was to discriminate between geographical areas linked to the dialects in 3 zones (Maghreb, Middle-East and Intermediate, i.e. Egypt and Tunisia), having achieved an area identification rate of $98 \%$.

Unfortunately, we do not have the same experience on Portuguese dialect iden- 
tification. In the next section, we will describe the main differences between the Portuguese varieties and discuss how we can take them into account in our system.

\section{Main differences between the varieties of Portuguese}

This section summarizes the main differences between some of the varieties spoken in CPLP countries (Community of Portuguese-speaking Countries). Portuguese is a language that is spoken by more than 170 million people in virtually all continents, ranking it very high among the most spoken languages in the world. The current work does not cover all of them, being restricted to the varieties to which we could have easy access in term of Broadcast News $(\mathrm{BN})$ recordings ${ }^{1}$ :

- European Portuguese (henceforth denoted as EP), the variety spoken in Portugal, for which the available speech recognition system has been trained.

- Brazilian Portuguese (henceforth denoted as BP), the variety spoken in Brazil, with the largest number of speakers.

- African Portuguese (henceforth denoted as AP), the generic name that covers all the varieties spoken in African countries that have Portuguese as official language (PALOP countries): Angola (AN), Cape Verde (CV), Guinea-Bissau (GB), Mozambique (MO) and São Tomé and Príncipe (ST).

Whereas there are already quite a few reports on the differences between EP and $\mathrm{BP}$, the differences between these varieties and AP are much less studied. Many of the comments made in this paper concerning AP will hence be made based on the study of the corpus described in Section 5. Unfortunately, Broadcast News is not the type of controlled conditions corpus that should ideally be used for this purpose. Speakers from African countries often have Portuguese as second language (namely in rural areas), and we cannot guess the native language in such multilingual environments. Their education degree is also very variable, as is the contact they may have with other varieties of Portuguese. Hence our comments on AP are mostly preliminary and need further corroboration with more controlled corpora.

\subsection{Orthographic and syntactic differences}

The current orthographic convention allows for minor differences, representing some phonetic and phonological specificities: the optional suppression of unpronounced consonants in BP (e.g. acção / ação, excepto / exceto), the optional use of hyphenation, and differences in diacritics (e.g. tranquilo / tranqüilo, accounting for the fact that $u$ is pronounced as $/ \mathrm{w} /$, instead of deleted as in the general case in-

$\overline{1}$ Speakers from Timor were unfortunately very scarce in BN transmitted in Portugal 
volving qui or que sequences; Jerónimos / Jerônimos, accounting for the different vowel quality).

Besides these differences, there are also significant ones concerning the use of prepositions, the position of clitics and the alternative use of infinitive/gerundive verb forms (e.g. estava sempre a meter-se em sarilhos vs.estava sempre se metendo em sarilhos - was always getting into trouble).

African countries that have Portuguese as official language follow the same orthographic conventions as for EP. Although the written form is very similar in AP and $\mathrm{EP}$, in spontaneous speech in AP one can find very frequent instances of lack of number agreement (e.g. os joelho instead of os joelhos 'the knees'). The causes for this phenomenon, which can also be found in BP, are controversial. Some authors relate it to the influence of Bantu languages, where the plural form does not need to be marked in both the determinant and the noun, as in the example above.

\subsection{Phonetic and phonological differences}

There is common agreement that one of the most striking differences between Brazilian and European varieties concerns vowel reduction, which is much more extreme in EP than in BP [25], [3]. EP unstressed high vowels are often deleted and rather long consonant clusters may surface within as well as and across word boundaries, which are not allowed in BP (e.g. se desprezarmos [sdfprz'armuf] 'if we ignore'). As empty nuclei are also obligatorily filled in BP, most two-obstruent sequences are broken by an epenthetic vowel (e.g. psicologia [pisikoloz'ir] 'psychology', afta ['afite] 'aphtha' in BP vs [psikluz'ie], ['afte] in EP). Loanwords can be treated rather differently, as well (eg. [izn'obi] in BP vs [sn'כb] in EP). Although both varieties distinguish between seven vowels in stressed position (/i e $\varepsilon$ a $ว$ o u/), they do not have the same reduction patterns, and quality changes are not sensitive to the same constraints.

The number of contrasting vowels is context dependent in BP: in pre-tonic position, $/ \mathrm{e} /-/ \varepsilon /$ and $/ \mathrm{o} /-/ \mathrm{\jmath} /$ contrasts are neutralised and the seven-vowel system reduces to the five-vowel system /i e a o u/, whereas in post-tonic position, it reduces to the three vowel system /i e u/, as /i e $\varepsilon /$ and / $\mathrm{u}$ o o/ merge to [i] and [u] respectively, and $/ \mathrm{a} /$ is raised to $[\mathrm{e}]$. EP does not show this type of variation, as its four-vowel system (/i ə e u/) holds for both positions.

In BP, unstressed vowels must also agree in height with the word stressed vowel (e.g. preferência (preference) [prefer'ẽsje] - preferível (preferable) [prifir'ivew]. Vowel height harmony in BP has been extensively studied, as it constitutes an important factor for the differentiation of BP dialects [5], [21]. According to these authors, it is a variable rule, which mainly affects the vowel immediately adjacent to the stressed one, and whose application depends on a multiplicity of factors (such 
as the presence/absence of a front vowel in the stressed syllable, presence/absence of a morphological boundary, speaker's age, etc.). Vowel lowering is typical of northern dialects and is practically nonexistent in Rio and S. Paulo. As for the raising of mid vowels, the authors found that harmony is respected in $32 \%$ and $29 \%$ only, for [e] and [o] respectively.

Although stressed vowels are rather similar in both varieties, there are some small differences worth mentioning. In EP, an additional vowel ([e]) may also appear in this context, as in some dialects including the Lisbon one:

(1) $[\mathrm{e}] /[\mathrm{a}]$ distinguish between the 1 st person plural of verbal present and past tense forms, respectively (e.g. pensamos (we think) [pẽs'emuf] / pensámos (we thought) [pẽs'amuf]);

(2) low vowels are raised before heterosyllabic nasal consonants (e.g. cara (face) [k'are] / cana (cane) [k'ene]);

(3) front vowels centralise before palatal consonants and glides (e.g. desenho (drawing) [dz'eju], telha (tile) [t'ê̌ ], lei (law) [l'ej]).

In BP the two forms in (1) are homophones (pensamos [pẽsemus] or [pẽsemuf]), and provided the orthography is correct the desired pronunciation is generated. In fact, although since [20], it has often been pointed out that nasalization is much stronger in BP than in EP, it has also been shown [1] that it is not a categorical rule: full nasalization is favored in stress position ( $>90 \%$ of the cases) but several factors, such as the presence of empty onsets or morphological boundaries, may inhibit nasal spreading in other contexts. On the other hand, some EP speakers may also strongly nasalize vowels in stressed position.

As for the main differences concerning the consonantal system, they are well known. In EP, coronal plosives are realized as [t] and [d], whereas in BP they are realized as [t $\left.\int\right]$ and [dz], respectively, before $/ \mathrm{i} /$.

Coda consonants in BP may considerably differ from EP ones in the same context. In fact, the realization of the so-called strong and weak "r's" varies considerably across the country, namely in coda position. In coda position, " 1 " is realized as [1] in EP, and as a labio-velar offglide, in BP. Due to this fact, a larger diphthong list can be found in BP.

In BP, diphthongs may also emerge from yodisation of some vowels followed by $/ \mathrm{J} /$, as in arroz (rice) [ar'oj $\left.\int\right]$.

Having summarized the main differences between EP and BP, which are fairly well studied, let us know address the much more unexplored comparison with AP varieties.

The multilingual background of many AP speakers may be the cause for the very large variability in the reduction patterns of AP varieties, both inter and intra- 
speaker. On one hand, one can find instances of vowel epenthesis in order to break consonant clusters and respect the $\mathrm{CV}$ syllable pattern. On the other hand, one can also find a generalization of EP reduction rules that, together with the influence of complex consonants of some native languages, may lead to patterns of vowel reduction even more extreme than those found in EP.

Similarly to BP, in AP consonant clusters formed across word boundaries may be solved in different ways: either by insertion of a paragogic vowel or by deletion of the coda consonant in the last syllable of the first word. In our data, the most common epenthetical vowel is /ə/, rather than /i/ as in BP. The latter occurs mostly in the last position of verbal forms ending in consonant. Contrarily to what is generally thought, there is no evidence that this vowel may be a copy of the following syllable nucleus. It is possible that, for other varieties such as observed for $\mathrm{MO}^{2}$, the process is very frequent for borrowings of Portuguese words by native languages but not to dissolve clusters in Portuguese words.

Contrarily to BP, in AP vowels are often deleted between nasals and obstruent consonants and pre-nasalized onsets often occur (e.g. amizade 'friendship', pronounced as [emz'adə] instead of [emiz'adə] as in EP). Deletion of high vowels or entire rhymes may also occur for AP within as well as across word boundaries, not only when the resulting sequences are similar to well-formed affricates, but also for other combinations of coronal fricatives with other obstruent consonants (e.g. psicólogo 'psychologist' often pronounced as [psk'olugu] in AP compared to [psik'olugu] in EP).

Concerning vowel reduction in pre-tonic position, a significant inter and intraspeaker variability is found in AP. Either there is vowel raising and centralization (sometimes more extreme than for EP) or there is a mixed behavior as some vowels are raised and others are not. This is often the case when a non-raised pre-tonic vowel would be produced with the same quality as the following stressed one.

Another very frequent phenomenon in AP is the neutralization of the $/ \mathrm{e} /-/ \varepsilon /$ and /o/-/o/ contrasts, but here, again, we have observed an enormous variability in all varieties.

The fact that some of the speakers do not contrast $/ \mathrm{e} /-/ \varepsilon /$ and $/ \mathrm{o} /-/ \mathrm{\jmath} /$ in stressed position and realize both vowels in each pair with an intermediate quality suggests that a contrast may not occur in their native languages. Apparently, there seems to be a generalization of an EP-metaphony rule according to which $/ \varepsilon /$ and $/ \mathrm{\jmath} /$ are realized as $/ \mathrm{e} /$ and $/ \mathrm{o} /$, respectively, in penultimate stressed open syllables, when the following syllable has a high rounded vowel (e.g. mesa 'table', pronounced as $\left.\left[\mathrm{m}^{\prime} \varepsilon \mathrm{z}\right]\right]$ in AP and as [m'eze] in EP; e.g. preso 'arrested', pronounced as [pr'ezu] in $\mathrm{AP}$ and $\mathrm{EP}])$.

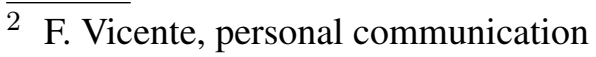


For all varieties but most noticeably in $\mathrm{CV}$, unstressed /a/ is generally pronounced as $/ \mathfrak{e} /$, even in closed syllables in which vowel reduction is blocked in EP (e.g. principalmente 'mainly', pronounced as [príspelm'ẽtə] in AP and as [prísipalñ'étə] in EP). Also, contrarily to EP, the fusion of two unstressed /a/ results in a single central vowel $[\mathrm{e}]$ instead of in a low one $([\mathrm{a}])$.

Falling diphthongs tend to monothonguize, in particular nasal ones, and what should be rising diphthongs in EP tend to be pronounced in hiatus. In the latter case, instead of the glide, a lowered vowel may be found (e.g. habituados, 'used', pronounced as [ebito'aduf] in AP and as [ebitw'aduf] in EP).

Coronal consonants are often apico-alveolar in all varieties. This is most noticeable for liquids. Some speakers do not produce a trill, neither in initial nor in intervocalic position.

\subsection{Prosodic differences}

The literature on the rhythm of Portuguese shows that there are controversial issues. In [29], for instance, EP is classified as stress-timed and BP as having mixed patterns of the syllable and stress-timed type. In [12], on the other hand, EP is characterized as having both stress-timing and syllable-timing properties, and BP as showing both syllable- and mora-timing properties. In a later paper [11], the same authors claim that EP and BP can be discriminated when the intonation pattern is preserved and all segmental information has been filtered out, and discuss the fact that intonation may be one of the important factors that lead to rhythmic distinctions, a topic that they view as worth pursuing.

Whereas comparative studies of BP and EP prosody can already be found (see also [10]), as far we know, such studies are inexistent for African varieties. However, we strongly believe that they will play a crucial role in distinguishing between themselves. In fact, the observation of our Broadcast News corpus allowed us to detect major differences between AP, BP and EP varieties from the segmental point of view, but these differences were more or less shared by all the AP varieties, with the above mentioned strong inter and intra-speaker variability. Subjects with some familiarity with the different African varieties are able to make a fair discrimination among them based on prosodic cues. The present work is a step towards studying these differences. 


\section{Language identification system}

After the necessarily brief review of the most recent LID approaches, we have retained the following options:

- The PPRLM systems seem to achieve the best results, so it is relevant to implement one. The main difficulty is that PPRLM systems need several highperformance phone recognizers. As there is already a phone recognizer available for the Portuguese language, and as our system is mainly targeted at testing the presence of the Portuguese language in $\mathrm{BN}$, we have decided to design a simple PRLM system using the Portuguese phone recognizer.

- The acoustic systems are an interesting compromise between complexity and performance. We have implemented a simple acoustic system using MFCC coefficients and Gaussian Mixture Models.

- As hypothesized by linguistic studies, prosody may also be a relevant cue to differentiate Portuguese varieties. Thus, taking in account our previous knowledge on prosody modeling and dialect identification [33,34], we have decided to implement a prosodic system, conjointly with the PRLM and acoustic system.

The system is thus a fusion of 3 subsystems: Acoustic (section 4.2), Phonotactic or PRLM (section 4.3), and Prosodic (section 4.4). These 3 subsystems use a common pre-processing module as represented in the Figure 1 . The pre-processing module will be briefly reviewed in the following section.

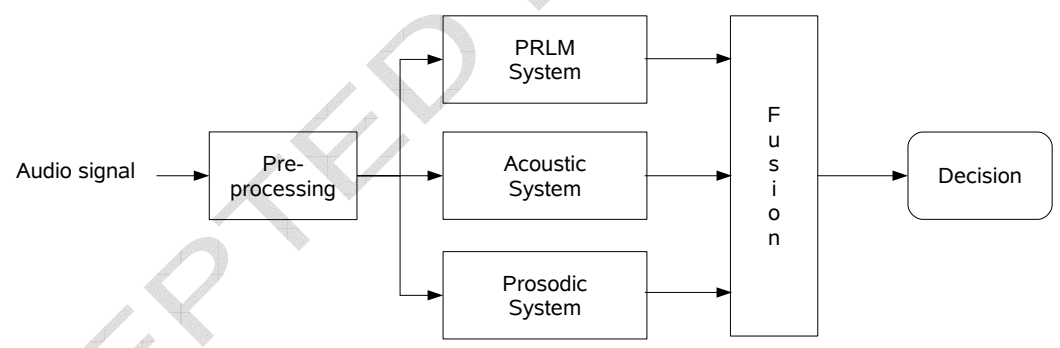

Figure 1. Overview of the language identification system.

\subsection{Audio pre-processing}

The language identification system is designed to be integrated in the speech recognition system. Therefore, it is relevant to take advantage of the audio pre-processing module also used in the speech recognition system. This module, developed by $\mathrm{H}$. Meinedo (see [27] and [28]), integrates five components (Figure 2): three modules 
for classification (Speech/Non Speech, Gender and Background), one for speaker clustering and one for acoustic change detection. All the modules are model-based, that is to say they use algorithms trained using a priori information. These models are composed of Artificial Neural Networks (ANNs) of the type feed-forward fully connected Multi-Layer Perceptron (MLP), and were trained with the backpropagation algorithm on a Portuguese BN corpus of over 60 hours.

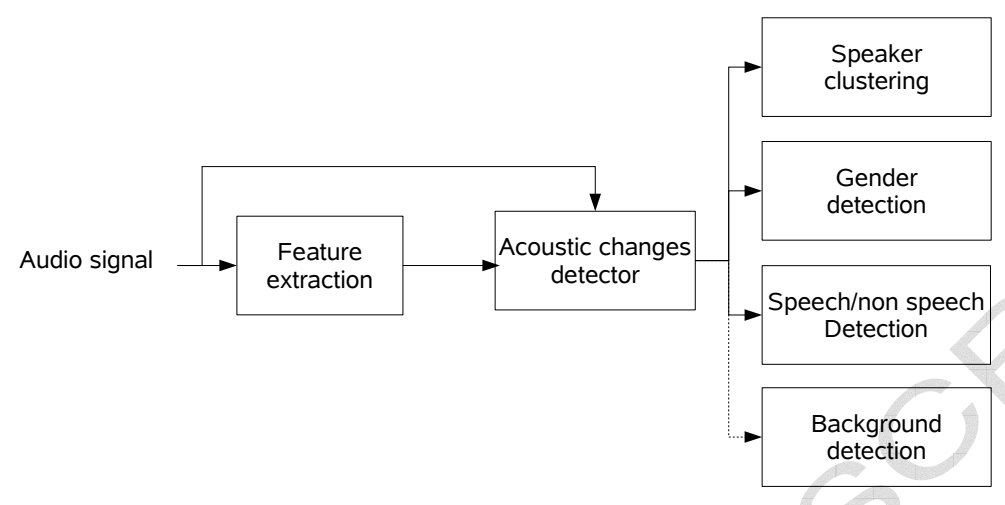

Figure 2. Overview of the audio pre-processing module.

Two of the modules of this pre-processing stage are specially interesting for language identification: the speech/non speech detection, as we do not want to treat non-speech parts, and the speaker clustering, as we assume that each speaker speaks a single language and make the language verification decision on a speaker by speaker basis.

All the modules were evaluated by $\mathrm{H}$. Meinedo on the COST 278 corpus described in section 5 . The acoustic change detector achieved a Recall value (\% of detected acoustic change points) of $78.9 \%$, a Precision value (\% of detected points which are genuine change points) of $65.5 \%$, and an F-measure (defined as $(2 *$ Recall $*$ Precision $) /($ Recall + Precision $))$ of 70.9 . The speech/non speech detector achieved an Accuracy of $95.6 \%$, and the gender detector of $94.5 \%$. Concerning the speaker clustering module, its performance was evaluated in terms of Q-measure $(68.1 \%)$ and Diarization Error Rate (DER=31.6\%). The Q-measure is defined as the geometrical mean of the percentage of cluster frames belonging to the correct speaker and the percentage of speaker frames labeled with the correct cluster and the DER is the percentage of frames with an incorrect cluster-speaker correspondence. As one speaker is often divided in several clusters, the performance in terms of DER is not very high, but this type of error does not affect the LID system.

Experiments on the Portuguese part of the COST 278 corpus have shown that using this pre-processing module has very little influence on the performance of the speech recognizer as compared to using manual speaker segmentation [28]. 


\subsection{Acoustic system}

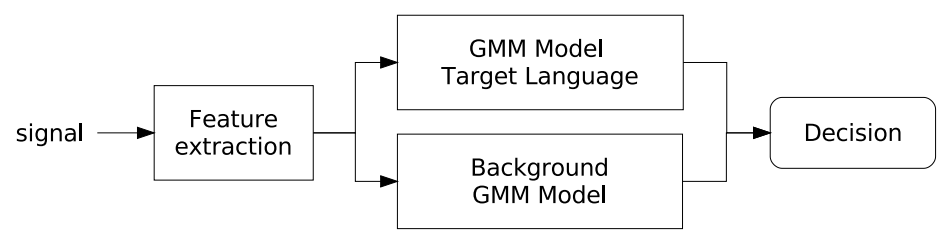

Figure 3. Generic acoustic language verification system.

A generic acoustic language identification system is displayed on Figure 3. The system works in two phases: a learning procedure to create the models, and a testing procedure. The acoustic features extracted from the audio signal are 12 MFCC plus delta, resulting in a 24-dimensional vector. The models used are Gaussian Mixture Models (as in [46]), learnt with the classic VQ and EM algorithms.

The background model is learnt using excerpts from all languages, while the target model is learnt using only the target language. No adaptation is used is this case. The verification test is made by comparing the likelihood of the test excerpt to the target-language model and to the background model.

Hence, the system output consist in a decision (true or false) if the language spoken in the test excerpt is the target language, and a confidence score (the ratio of the likelihoods from the target language model and the background model).

\subsection{PRLM system}

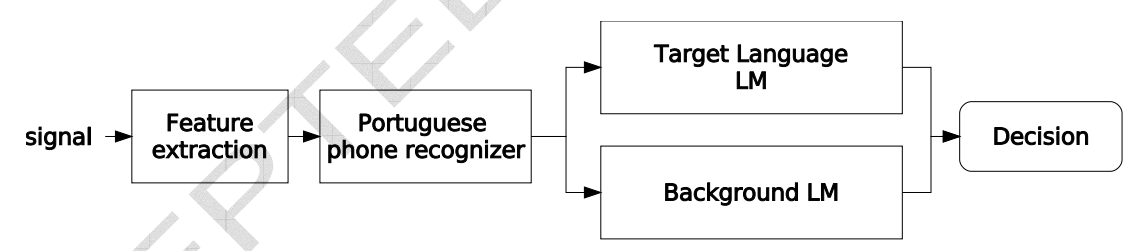

Figure 4. PRLM System overview.

As explained above, the PRLM system is based on a single Portuguese phonerecognizer (see [47] for a description of PRLM systems). A synoptic of the system is given in the Figure 4.

The phone recognizer is part of the AUDIMUS system [26]. AUDIMUS is a hybrid system that combines the temporal modeling capabilities of Hidden Markov Models (HMMs) with the pattern discriminative classification abilities of Multi-Layer Perceptrons.

The phonetic decoding in the AUDIMUS system is based on MLP models, trained on the above mentioned EP broadcast news corpus of over 60 hours. It combines 
phone probabilities computed from several MLPs using different feature sets: PLP (1 $12^{\text {th }}$ order plus delta), log-RASTA (12 ${ }^{\text {th }}$ order plus delta), and Modulation Spectrogram (MSG - 28 coefficients). The outputs of all 3 MLP classifiers are then merged using an average in the log-likelihood domain.

This phonetic decoding is applied to all the languages in the training database, resulting in Portuguese-phones sequences which are then modeled for each language by n-grams, using the SRI-LM toolkit [38]. A background n-gram model is also trained using data from all languages.

The verification test is made by comparing the likelihood of the test excerpt to the target-language model and to the background model. During the test phase, the identified language is defined according to the n-gram model providing the maximum of likelihood.

Like the acoustic system, the PRLM system output consists of a decision (true or false) if the language spoken in the test excerpt is the target language, and a confidence score.

\subsection{Prosodic system}

The prosodic system is the same as used in [33]. It is based on two different aspects: the definition of relevant units (pseudo-syllables) and the separate processing of the variations of macro- and micro-prosodic components (long- and short-term models). A synoptic of the system is displayed on Figure 5.

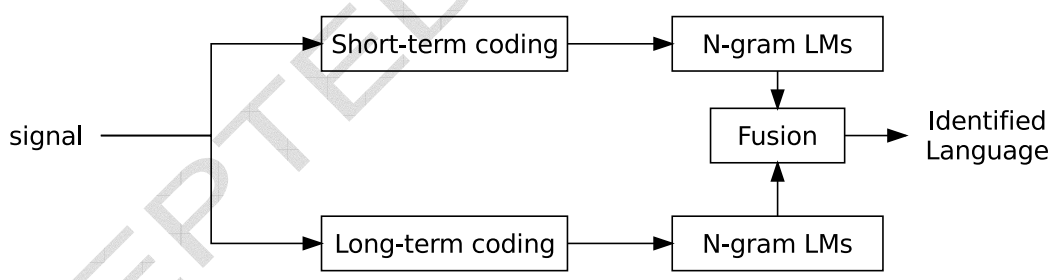

Figure 5. Prosodic system overview.

The pseudo-syllable unit is defined as a cluster of consonants ending with a vowel, corresponding to the most frequent syllable structure in the world [9]. Three baseline procedures lead to relevant consonant, vocalic and silence segment boundaries:

- Automatic speech segmentation based on the Forward-Backward Divergence" (DFB) algorithm [2], leading to infra-phonemic quasi-stationary segments.

- Vocal activity detection based on a first order statistic analysis of the energy signal [30].

- Vowel localization based on a spectral analysis [30]. 
This front-end processing results in a segmentation into vocalic, consonantal and silence segments. Labels "V", "C", or "\#” are used to qualify each segment, respectively. The next stage is pseudo-syllable gathering: all the consonantal segments are merged until the next vocalic segment, which ends the pseudo-syllable. Figure 6 shows the automatic segmentation and labeling results and the identified pseudo-syllables.

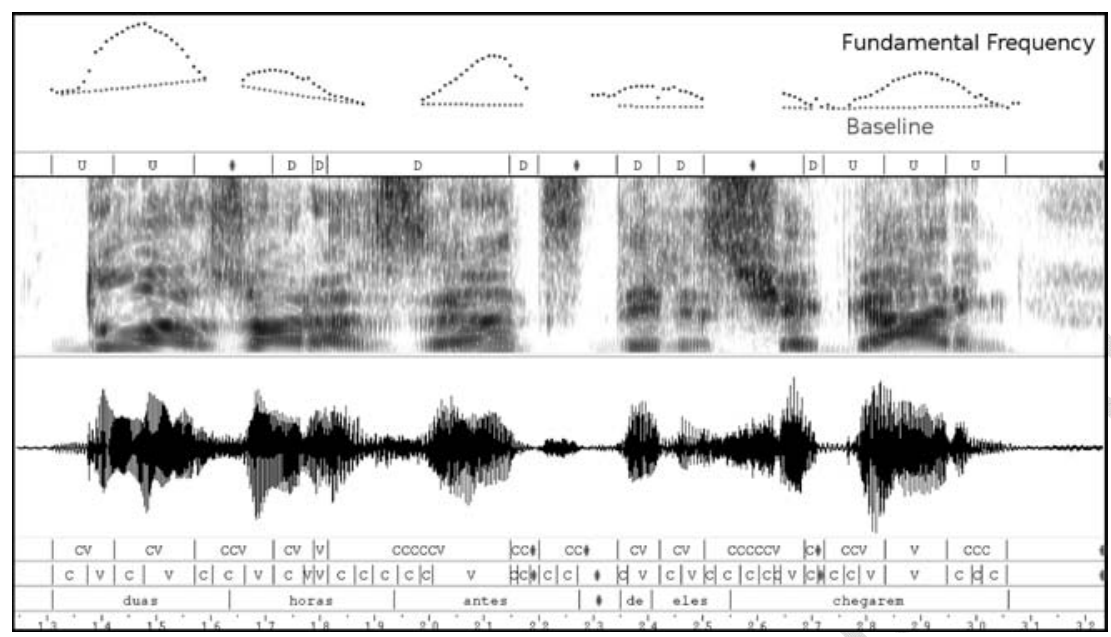

Figure 6. Spectrogram, signal representation and prosodic processing example for the sentence "na mesma noite duas horas antes de eles chegarem uma casa havia sido assaltada na cidade". Transcriptions are (from bottom to top): (a) manual word annotation, (b) automatic segmentation and labeling, (c) pseudo-syllables, and on top of the spectrogram: long-term coding.

Two models are used to separate the long-term and short-term components of prosody. The long-term component characterizes prosodic movements over several pseudo-syllables, while the short-term component represents prosodic movements inside a pseudo-syllable. The fundamental frequency processing is divided into two phases, representing the phrase accentuation and the local accentuation, as in Fujisaki's work [13]. The phrase accentuation is used for the long-term model while the local accentuation is used for the short-term model. Fundamental frequency and energy are extracted from the signal using the SNACK Sound toolkit [37].

The long-term coding uses the pseudo-syllable segmentation as a time-base. The coding is described in Figure 7. The "baseline" is a representation of the phrase

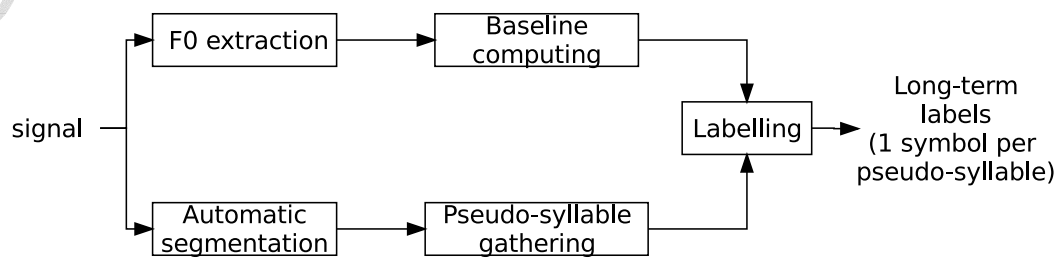

Figure 7. Long-term coding.

accentuation. It is computed by finding all the local minima of the $F_{0}$ contour, and 
linking them. The labels used are $\mathrm{U}(\mathrm{p}), \mathrm{D}(\mathrm{own})$, respectively representing a positive and a negative slope of the baseline, and \#(silence or unvoiced). An example of a resulting baseline curve is displayed in Figure 6.

The short-term coding is detailed on Figure 8. The short-term coding use the "C",

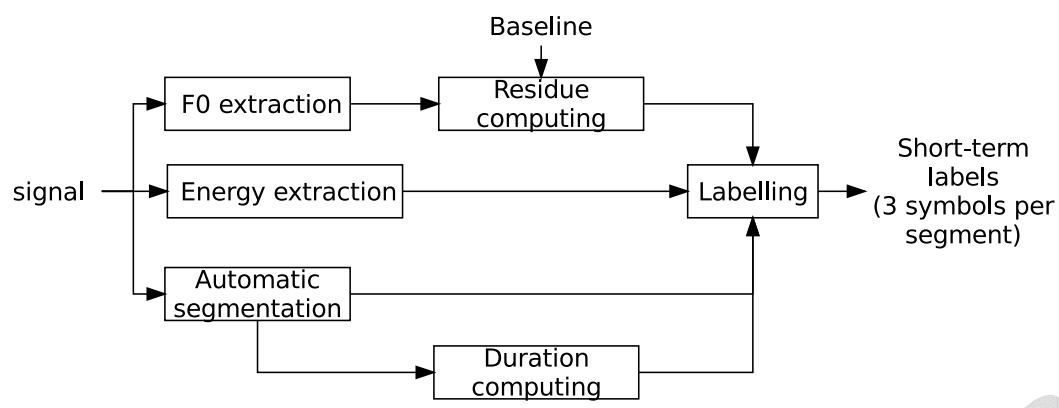

Figure 8. Short-term coding.

"V" and "\#" segments as a time base. The local accentuation, named here residue, is represented by the difference between the original $F_{0}$ contour and the baseline. This residue is then approximated on each segment by a linear regression. The F0 variation on voiced parts gives the label (Up or Down). Unvoiced parts are labelled "\#”. In parallel, the energy curve is computed and also approximated by linear regressions on each segment. The process is the same as the one used for the residue coding. The Up and Down labels are used to describe the variations while very short segments (e.g. <20ms) are labelled “\#”. Duration labels are also computed on the segment units. The "s" (short) and "l" (long) labels are assigned considering the mean duration of each kind of segment (vocalic, consonantic or silence). These three coding labels are used conjointly to form the short-term coding. Hence, for each segment, the label is then composed of three symbols.

To model the prosodic variations, we use classical n-gram language modeling provided by the SRI language modeling toolkit [38]. For each system - long- and short-term - each target language is modeled by a n-gram model during the learning procedure. A background model is also learned using data from all languages. During the test phase, the most likely language is picked according to the model (target or background) which provides the maximum likelihood. Several lengths for the n-gram models have been tested (from 3- to 5-grams), the best results are obtained with 3-grams on different kinds of databases [33].

\subsection{Fusion}

For the time being, the fusion method is only a simple weighted addition of the loglikelihoods generated by each system. The weights have been computed on the train part of the corpus described in the next chapter. The method is clearly non-optimal. 
Hence, it will not be described in detail and is only mentioned to give an idea of the performances that could be achieved using the three subsystems together in the section on experimental results (section 6).

\section{Corpora}

Two different corpora have been used for the experiments. The first corpus is used for the language verification experiment, i.e. to test the reliability of the language identification system, especially for Portuguese broadcast news. The second corpus is used for variety identification.

\subsection{Language Verification Corpora}

The COST 278 corpus was constructed by seven institutions that collaborated in the European action on Spoken Language Interaction in Telecommunications ${ }^{3}$. It comprises broadcast news shows in nine languages, namely Dutch (from Belgium, noted BE), European Portuguese (EP), Galician (GA), Czech (CZ), Sloven (SI), Slovakian (SK), Greek (GR), Croatian (HR) and Hungarian (HU) [41]. The first part of Table 1 shows the countries and languages used by the different TV stations, the number of collected shows and the total data size (in minutes).

Since there were no English recordings in this corpus, and given the fact that English is the most frequent language, next to Portuguese, found in Portuguese broadcast news, we complemented the COST 278 corpus with a subset of the 1996 Broadcast News Speech Corpus. This corpus contains a total of 104 hours of broadcasts from $\mathrm{ABC}, \mathrm{CNN}$ and CSPAN television networks and NPR and PRI radio networks with corresponding transcripts. The primary motivation for this collection was to provide training data for the DARPA "HUB4" Project on continuous speech recognition in the broadcast domain.

For the purpose of our language identification studies, we only used the first data $\mathrm{CD}$ in order to keep consistency with the amount of data available in the COST 278 corpus. The programs used in our experiments are 10 shows from "ABC Nightline", with a mean duration of approximately $30 \mathrm{~min}$. The corresponding information is shown in the bottom part of Table 1.

$\overline{3 \text { http }: / / \operatorname{cost} 278 . \text { org/ }}$ 
Table 1

Overview of the COST 278 corpus (top part) complemented with the subset of the HUB4 corpus (bottom).

\begin{tabular}{lllcc}
\hline Code & Country & Language & \# of shows & Duration (min) \\
\hline BE & Belgium & Dutch & 6 & 150 \\
CZ & Czech Rep. & Czech & 5 & 171 \\
GA & Spain & Galician & 4 & 184 \\
GR & Greece & Greek & 3 & 174 \\
HR & Croatia & Croatian & 6 & 166 \\
HU & Hungary & Hungarian & 11 & 166 \\
EP & Portugal & Portuguese & 6 & 190 \\
SI & Slovenia & Sloven & 3 & 151 \\
SK & Slovakia & Slovak & 9 & 165 \\
\hline EN & United States & English & 10 & 328 \\
\hline Total & 10 & 10 & 66 & $33 \mathrm{~h} 47 \mathrm{~min}$ \\
\hline
\end{tabular}

\subsection{Train and test sets}

Train and test sets have been defined for each language. The test set contains one or two shows per language. The remaining shows are used in the train set.

The train set contains a total of 1659 automatically detected speech segments, for a total duration of 16 hours and 12 minutes. The duration per language ranges from 114 minutes to 168 minutes.

The test set has a total duration of 7 hours 15 minutes, with 789 automatically detected speakers. The duration per language ranges from 24 minutes to 75 minutes.

\subsection{Variety Verification Corpora}

\subsubsection{European Portuguese}

For the variety verification task, we used the EP subset of the COST 278 corpus. Since this corpus includes different varieties of Portuguese, it was manually processed to eliminate the non-EP speakers. The duration is now 152 minutes, for the train set, and 21 minutes for the test set.

The short duration of the EP test set, relative to what became available for other varieties, led us to add an extra daily news show. The new EP corpus has a total of 
230 minutes (78 for testing), and 336 automatically detected speakers.

\subsubsection{Brazilian Portuguese}

The Brazilian recordings come from news shows of the TV Record Brazilian channel. We have recorded 12 shows, ranging from 20 to 50 minutes each. After preprocessing, we have a total of 367 minutes of Brazilian speech data, with 452 automatically detected speakers.

\subsubsection{African Portuguese}

Reporter Africa is the main news programs from the RTP Africa channel. Each daily show lasts for around 25 minutes, with information from reporters in Angola, Cape Verde, Guinea-Bissau, Mozambique, São Tomé and Príncipe. The anchor speaks European Portuguese. We have recorded 24 shows and labeled the varieties for each of these. The total duration is $10 \mathrm{~h}$, but we have excluded the EP speakers, foreign speakers and also the few speakers for which the human annotators could not distinguish the country of birth, being only able to tell they were from Africa. The number of speakers and the duration (in minutes) for each African variety is shown in Table 2.

Table 2

Portuguese varieties recordings from "Reporter África".

\begin{tabular}{clcc}
\hline Code & Country & \# speakers (test) & Duration in minutes (test) \\
\hline AN & Angola & $84(35)$ & $71(23)$ \\
CV & Cape Verde & $116(24)$ & $78(11)$ \\
GB & Guinea-Bissau & $70(26)$ & $71(22)$ \\
MO & Mozambique & $77(22)$ & $77(23)$ \\
ST & São Tomé and Príncipe & $83(31)$ & $62(18)$ \\
\hline Total & 5 & $430(138)$ & $359(97)$ \\
\hline
\end{tabular}

\subsubsection{Train and Test sets}

Preliminary experiments with a reduced data set were first carried out using a crossvalidation procedure [35], given the relative low volume of data for each variety. First, one speaker was selected for testing. All the remaining data was used for learning the variety models. After the test was completed, a new speaker was used for testing. This procedure was iterated until all the speakers of the corpus have been used for testing. The problem with this first approach was that speakers were 
clustered independently for each show and we did not guarantee that the same speaker was not used both for training and testing.

The current data set is roughly $70 \%$ larger, which enabled us to have separate train and test sets. In selecting these sets, we tried to guarantee that the same speaker was not present in both train and test sets. The percentage of the corpus used for testing ranged from $14 \%$ to $34 \%$ for all the varieties.

\section{Language Verification Experiments}

The language verification results shown in this chapter were computed using the verification framework adopted in the recent NIST evaluation compaigns. Results include miss and false alarm probabilities, DET curves ${ }^{4}$, and Equal Error Rates.

In the language verification corpus, we have a total number of 789 test speakers. Considering that we test the detection for all the 10 languages, we have a total of 7890 language verification tests, with 789 target trials and 7101 non-target trials. The results are displayed in the Figure 9.

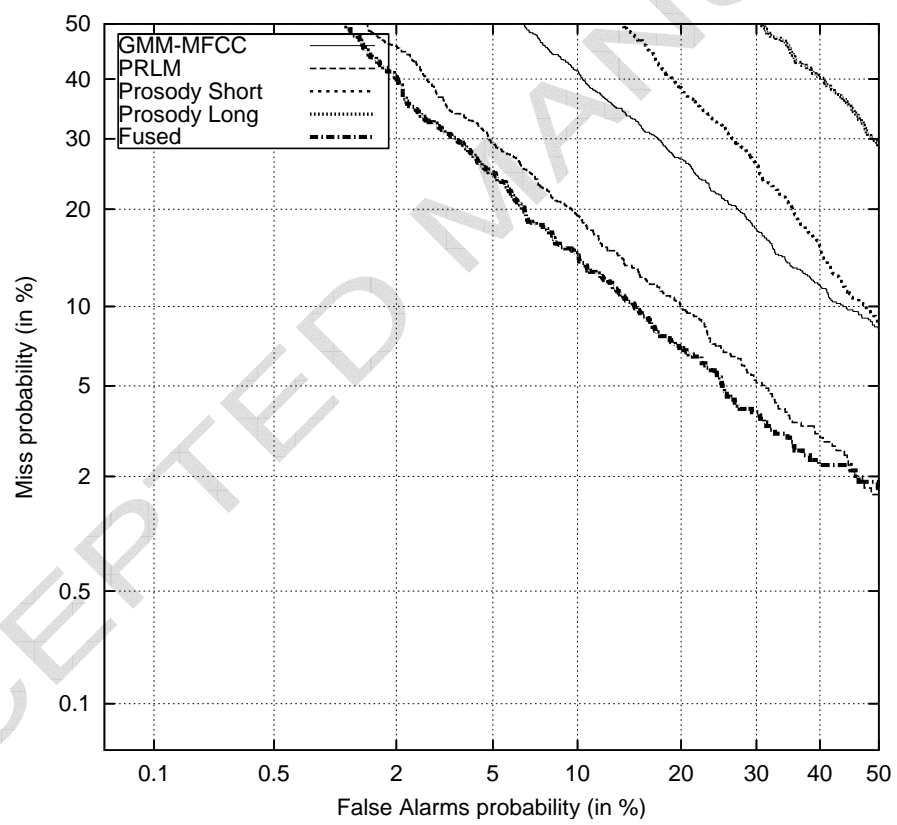

Figure 9. DET curve obtained using all the systems.

The best results are unsurprisingly given by the PRLM system, using 3-grams. The GMM-MFCC system gives the second best results, followed by the prosodic shortterm system. The DET curve obtained using the fused system is also displayed in Figure 9. Results are given in terms of EER in Table 3, for each system and each language. Different thresholds are used for computing the EER for each language.

4 http://www.nist.gov/speech/tools/ 
Table 3

Results per language in terms of \% EER.

\begin{tabular}{cccccccccccc}
\hline Language & BE & CZ & EN & GA & GR & HR & HU & EP & SI & SK & ALL \\
\hline Fused (\% EER) & 3.6 & 13.8 & 5.3 & 6.6 & 19.7 & 9.1 & 5.2 & 2.5 & 19.0 & 18.1 & 12.4 \\
\hline
\end{tabular}

The overall performance of the fused system is $12.4 \%$ EER. The worst performance is obtained for Greek with $19.7 \%$ EER, while the best performance is $2.5 \%$ EER for Portuguese. This is not at all surprising, given the use of the Portuguese phone recognizer in the PRLM system. The fused system also achieves good performance for Belgian Dutch (3.6\% EER), English (5.3\% EER) and Hungarian (5.2\% EER). Tests with much larger corpora should be made to evaluate if such differences in performance are significant.

Tables 4 and 5 show respectively the number of false alarms and missed detections over all languages and, given the focus of our work, for Portuguese. These results were obtained using the fused system.

Table 4

False detections for the fused system.

\begin{tabular}{cccc}
\hline Language & \# false alarms & \# non-targets trials & \% EER \\
\hline EP & $\mathbf{1 9}$ & $\mathbf{7 5 0}$ & $\mathbf{2 . 5}$ \\
\hline ALL & 888 & 7101 & 12.5 \\
\hline
\end{tabular}

Table 5

Missed detections for the fused system.

\begin{tabular}{cccc}
\hline Language & \# Missed detections & \# targets trials & \% EER \\
\hline EP & $\mathbf{1}$ & $\mathbf{3 9}$ & $\mathbf{2 . 6}$ \\
\hline ALL & 98 & 789 & 12.4 \\
\hline
\end{tabular}

The Portuguese false alarms are distributed across the different languages: 1 from English, 9 from Galician, 5 from Greek, 3 from Sloven, and 1 from Slovak. As Galician and Portuguese are closely related, it is not surprising to find that some Galician speakers are identified as Portuguese. All the errors are linked either to bad acoustic conditions (e.g. live sports reports) or very short test segments (e.g. duration under 5 seconds).

The only missed detection error for the Portuguese verification system appears on a 2 second segment, which is in fact a music segment in English, wrongly labeled as speech by the pre-processing module.

As a result from this analysis, we can hypothesize that the acoustic environment 
and the short length of the test segments, combined with pre-processing errors, are the main factors that lead the system to generate errors, at least for the Portuguese language. This behavior seems however to be the same for all languages. Since the detection of the acoustic environment is one of the tasks of the pre-processing module (see section 4.1), we will take advantage of the current work towards its improvement.

\subsection{Impact of the test segment duration}

As the duration of the test segment varies greatly, we have investigated how the performance of the system increases when discarding very short segments. These experiments show how the different systems work with segments with minimum length of $10 \mathrm{~s}, 20 \mathrm{~s}$, and $30 \mathrm{~s}$.

Table 6

Results for all the systems in terms of \% EER depending on the minimum duration of the test segments.

\begin{tabular}{ccccc}
\hline Min. duration & $0 \mathrm{~s}$ & $10 \mathrm{~s}$ & $20 \mathrm{~s}$ & $30 \mathrm{~s}$ \\
\hline \# test segments (ALL) & 789 & 618 & 394 & 272 \\
\hline ALL (\% EER) & 12.4 & 9.3 & 6.6 & 5.8 \\
\hline EP (\% EER) & 2.5 & 0.2 & 0.00 & 0.00
\end{tabular}

The first line of Table 6 shows that the number of test segments reduces (for all languages) when only long segments are selected. As expected, the performance of the system shown in the second line improves when using longer segments. The improvement is clearly significant when selecting segments of duration over 30s: the EER becomes $5.8 \%$ instead of $12.4 \%$. This is illustrated by the DET-curve displayed on Figure 10. Selecting segments that have enough information for identifying the language is clearly needed to achieve better performance.

The same type of analysis is shown in particular for the Portuguese language verifier in the last line of Table 6 . One can observe that the system does not make any errors for files over 20 seconds, and that the error rate is only $0.2 \%$ for files over 10 seconds. As the error rate seems sufficiently low for the Portuguese verification task, the next step is to investigate how this system behaves when trying to identify the different varieties of Portuguese - European, Brazilian, and African. 


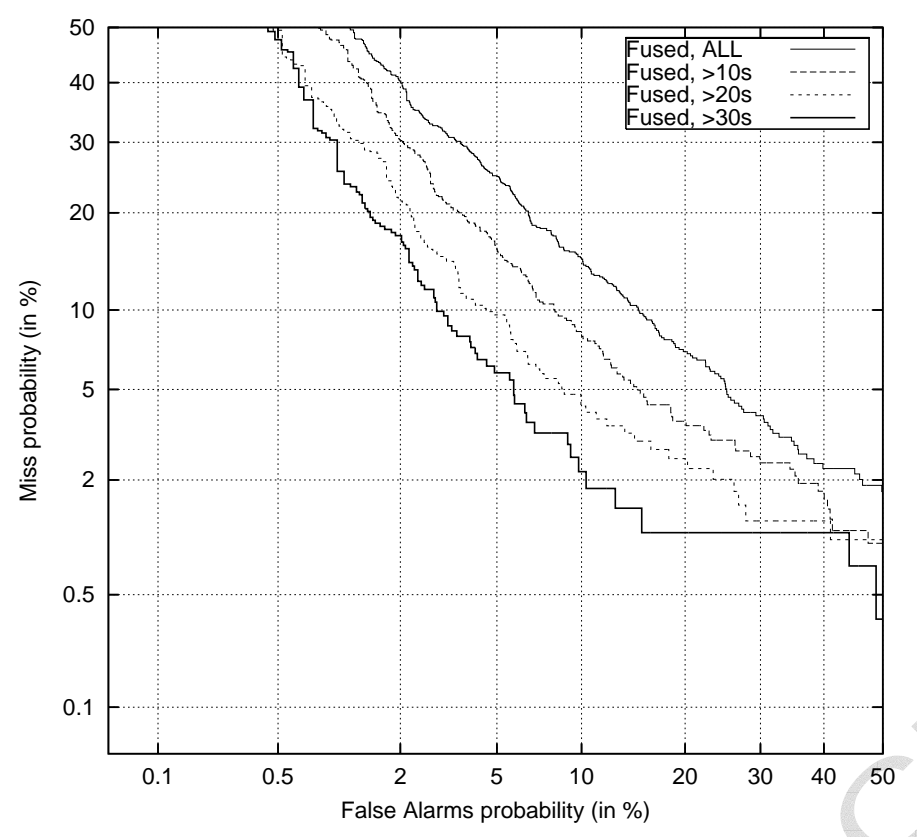

Figure 10. DET curve obtained using the fused system.

\section{Behavior of the language verifier with other varieties of Portuguese}

The aim of this experiment is to investigate how the Portuguese language verifier behaves when confronted with data from all the varieties of Portuguese. In the experiments described above, we only have considered European Portuguese. For this experiment, we only used the Portuguese language verifier. The test data described in section 5.3 is used for testing - Thus, we have all African varieties speakers (138) plus Brazilian speakers (147) plus European Portuguese speakers (125). What is expected is that this data should be recognized as Portuguese (as opposed to the other languages: English, Dutch, etc.), leaving the possibility of a second classification phase designed to detect the Portuguese variety.

Table 7 shows the results obtained for all the segments durations. When not selecting a minimum length, there is a total of 410 segments to test. The number of test segments becomes 228 when considering segments of duration superior to 10 seconds, 152 for segments superior to 20 seconds, and 94 for segments superior to 30 seconds.

Table 7

Results for the system in terms of \% EER depending on the minimum duration of the test segments.

\begin{tabular}{ccccc}
\hline Min. duration & $0 \mathrm{~s}$ & $10 \mathrm{~s}$ & $20 \mathrm{~s}$ & $30 \mathrm{~s}$ \\
\hline \# test segments & 410 & 228 & 152 & 94 \\
\hline Fused system (\% EER) & 20.7 & 16.6 & 13.8 & 10.6 \\
\hline
\end{tabular}


The variety which is least recognized as Portuguese is Brazilian Portuguese, which is responsible for all the errors of the system. A closer look at the errors allows us to see that most occur during the headlines or weather forecasts, which contain loud background music.

\section{Discrimination between Portuguese varieties}

Given that the amount of data for each variety is not very well balanced (much more data for the European and Brazilian varieties than for each separate African variety), we first tried to address the problem of identifying 3 broad varieties by regrouping all the African varieties into one class. Thus, the aim of this experiment is to verify if the test speaker speaks African, Brazilian or European Portuguese. The designed system performs fairly well on this data, with a global identification rate of $83.9 \%$. Detailed results (Table 8 ) show that the best identified variety is Brazilian Portuguese (96.6\%). This result is obtained using the fused system.

Table 8

Identification of Portuguese varieties - Confusion matrix using only 3 broad classes (African, Brazilian and European Portuguese). \% correct $=83.9 \% \pm 3.5 \%$.

\begin{tabular}{cccc} 
& AP & BP & EP \\
\hline AP & 79.7 & 12.3 & 8.0 \\
BP & 3.4 & 96.6 & 0.0 \\
EP & 14.4 & 12.0 & 73.6
\end{tabular}

African varieties tend to be confused with BP and, although no so often with EP. The next experiment aims at assessing the confusability between African varieties themselves. The global identification rate (see Table 9) is only $42.0 \%$. The most clearly identified variety is the one from Cape Verde.

\subsection{Human benchmark experiment}

In order to compare the performance of our automatic variety identification system with a manual one, we conducted a human benchmark. For this purpose, we have selected 8 stimuli from each of the 7 varieties. In this selection, we avoided sentences that could give an indication either by lexical, syntactical or semantical cues of the origin of the speaker. That is, we avoided the mention of locations, politicians, political parties, etc.. We also avoided sentences with clitics, since the 
Table 9

Identification of African Portuguese varieties - Confusion matrix produced by the fused system. $\%$ correct $=42.0 \% \pm 8.2 \%$

\begin{tabular}{cccccc}
\hline & AN & CV & GB & MO & ST \\
\hline AN & 31.4 & 20.0 & 5.7 & 20.0 & 22.9 \\
CV & 4.2 & 66.7 & 8.3 & 12.5 & 8.3 \\
GB & 3.8 & 42.3 & 30.8 & 15.4 & 7.7 \\
MO & 13.6 & 13.6 & 13.6 & 59.1 & 0.0 \\
ST & 9.7 & 29.0 & 3.2 & 25.8 & 32.3 \\
\hline
\end{tabular}

Brazilian origin would be very noticeable, and sentences where the lack of number agreement would make the African origin too noticeable. In this way, the human benchmark test was made in conditions as close as possible to the ones of our automatic variety identification system. The sentences (or segments from sentences) ranged in duration between 1.6 and 23.4 seconds. Most of the sentences were extracted from spontaneous speech $(64 \%)$, in order to avoid easily identifiable journalists or politicians. In addition to the 8 sentences, the participants were asked to identify the variety of 2 words (also extracted from sentences). The total duration of all stimuli was 8.5 minutes. Participants were asked to classify each stimulus as one of the 7 varieties, but they also had an option to mark it as African Portuguese (AP). In very few cases they forgot to (or could not) mark their preference (no answer NA).

The test involved 65 participants, currently living in Portugal. 44 participants were Portuguese, 7 were from Brazil and 14 from Africa (8 from Angola, 4 from Cape Verde and 2 from Mozambique).

Table 10 shows the confusion matrix results of this test, with a dividing line between sentences (top part) and words (bottom part).

- The results very clearly show that, as in the automatic test, Brazilian Portuguese is the least confusable variety. They also show that European Portuguese is next and that African varieties are easily confused with each other. Among these varieties, ST was the hardest to identify. The results with words were naturally inferior, except for BP, showing a greater tendency towards classifying African varieties as AP.

- It was interesting to notice that practically all Portuguese participants correctly identified BP and (although not so clearly) EP sentences, and most could correctly identify African varieties as such but, even if they have some suspicion about the African country of origin, namely if they have lived there, they were often reluctant to discriminate. 
Table 10

Human benchmark results (\% of correct identification). The top part shows the results with sentences and the bottom part shows the results with words.

\begin{tabular}{cccccccc|cc}
\hline Variety & AN & BP & CV & EP & GB & MO & ST & AP & NA \\
\hline AN & 20.0 & 0.6 & 7.5 & 0.0 & 7.3 & 9.2 & 8.1 & 47.3 & 0.0 \\
BP & 0.0 & 99.2 & 0.4 & 0.0 & 0.0 & 0.0 & 0.2 & 0.2 & 0.0 \\
CV & 11.0 & 0.4 & 16.5 & 4.8 & 4.0 & 10.4 & 6.7 & 45.8 & 0.4 \\
EP & 1.9 & 0.6 & 1.3 & 88.7 & 0.4 & 1.0 & 0.8 & 5.4 & 0.0 \\
GB & 17.7 & 0.2 & 8.3 & 2.1 & 10.0 & 8.7 & 7.7 & 45.2 & 0.2 \\
MO & 13.7 & 0.2 & 5.4 & 1.5 & 7.7 & 14.6 & 9.4 & 47.1 & 0.4 \\
ST & 14.4 & 1.2 & 10.4 & 2.5 & 8.1 & 10.2 & 9.2 & 43.8 & 0.2 \\
\hline AN & 20.8 & 0.0 & 2.3 & 0.8 & 3.1 & 6.9 & 5.4 & 60.0 & 0.8 \\
BP & 0.0 & 99.2 & 0.0 & 0.0 & 0.0 & 0.0 & 0.0 & 0.0 & 0.8 \\
CV & 4.6 & 1.5 & 12.3 & 3.1 & 6.2 & 3.1 & 4.6 & 63.8 & 0.8 \\
EP & 1.5 & 1.5 & 4.6 & 73.8 & 0.0 & 3.8 & 1.5 & 13.1 & 0.0 \\
GB & 10.0 & 0.0 & 3.8 & 3.8 & 6.2 & 11.5 & 6.9 & 57.7 & 0.0 \\
MO & 10.0 & 0.0 & 4.6 & 7.7 & 8.5 & 7.7 & 5.4 & 56.2 & 0.0 \\
ST & 12.3 & 0.0 & 5.4 & 11.5 & 2.3 & 7.7 & 4.6 & 55.4 & 0.8 \\
\hline
\end{tabular}

- Some Brazilian participants had no familiarity at all with African varieties, tending to confuse them with EP.

- Most African participants correctly identified BP and EP varieties, but they also tried to discriminate between African varieties more often. Their general opinion was that identifying African varieties in $\mathrm{BN}$ was much more difficult than identifying the varieties of the African people they meet everyday, most probably because in BN, many speakers (reporters, politicians and people involved in cultural events) have a higher level of education and/or familiarity with EP.

If these results are analyzed using only three broad classes (AP, BP and EP), as shown in Table 11, the average ratio of correct identification is $96.2 \%$ for sentences and $91.8 \%$ for words.

Just for comparison purposes, we have also run an experiment aimed at investigating the behavior of the automatic variety identification system with these stimuli. The number of files is too small to get any significant results, and some of the files were too short, but still the automatic 3-class system yielded reasonably good results (above 70\%). 
Table 11

Human benchmark results with only 3 broad classes. The top part shows the results with sentences (correct $=96.2 \%$ ) and the bottom part shows the results with words (correct $=91.8 \%$ ).

\begin{tabular}{ccccc}
\hline Variety & AP & BP & EP & NA \\
\hline AP & 97.1 & 0.5 & 2.2 & 0.2 \\
BP & 0.8 & 99.2 & 0.0 & 0.0 \\
EP & 10.8 & 0.6 & 88.7 & 0.0 \\
\hline AP & 93.8 & 0.3 & 5.4 & 0.5 \\
BP & 0.0 & 99.2 & 0.0 & 0.8 \\
EP & 24.6 & 1.5 & 73.8 & 0.0 \\
\hline
\end{tabular}

\subsection{Automatic speech recognition experiments}

It is also interesting to relate the results of the automatic/human variety identification tests with the results obtained with an automatic speech recognition system trained for Broadcast News in EP. The acoustic models of this system have already been described in Section 4.2. The vocabulary includes around 57k words. The lexicon includes multiple pronunciations, totaling $65 \mathrm{k}$ entries. The corresponding outof-vocabulary (OOV) rate is $1.4 \%$. The language model, which is a 4-gram backoff model, was created by interpolating a 4-gram newspaper text language model built from over $604 \mathrm{M}$ words with a 3 -gram model built on around 532k words of manually transcribed broadcast news $(\approx 50$ hours). The language models were smoothed using Knesser-Ney discounting and entropy pruning. The perplexity obtained in a development set is 112.9.

Table 12 shows the ASR results in terms of word error rate (WER), obtained using all the training/test material of our accent identification system. The best performance was obtained for EP, obviously. The fact that the acoustic phones used in the PRLM module were the same as in the ASR module justifies the best performance of PRLM for this variety. The percentage of spontaneous speech in this subset is relatively low, which may also account for the low WER obtained. ${ }^{5}$ The worst performance was obtained for BP, a fact that was also expected given that it was so easily distinguishable from EP, both manually and automatically. Intermediate results were obtained for all African varieties, with very close WER values slightly above $40 \%$ for all of them. The OOV rate for Brazilian and African varieties is not significantly higher than the one obtained for EP (1.8\% for BP and $2.0 \%$ for AP) thus not being responsible for the large performance degradation.

\footnotetext{
$\overline{5}$ In other sets with a percentage of spontaneous speech closer to $40 \%$, the WER goes up to $23.5 \%$.
} 
Table 12

Word error rate results obtained on the multi-variety corpus by an EP-trained ASR system.

\begin{tabular}{cccccccc}
\hline Variety & AN & BP & CV & EP & GB & MO & ST \\
\hline \% WER & 42.8 & 73.5 & 43.0 & 19.8 & 42.7 & 40.6 & 44.3 \\
\hline
\end{tabular}

\section{Conclusions and Future Work}

The first part of this paper described a language verification system for Broadcast News. The system is composed of three modules used to model language discriminative features: phonotactics, acoustics and prosody. Over all the 10 languages of the multilingual $\mathrm{BN}$ corpus we have used, the average performance of the fused system is $12.4 \%$ EER. The comparison with other systems is not straightforward, since there is not so much reported work on Broadcast News data, and none with as many languages as we have used. The EER obtained with the fused system on the "segments over 30s" condition (5.8\%) may be compared to the best results obtained on the NIST 2005 data (4.2\% EER). The corpora used in both evaluations are, however, quite different. The NIST 2005 data is telephone speech, which is likely to have worse quality than broadcast news, but does not include so much diversity in terms of acoustic conditions, prepared and spontaneous speech, etc.. In fact, one of the approaches we are currently investigating in order to improve our system is to take into account these different acoustic conditions. Another difference between the two corpora lies in the constraints on the homogeneity of the segments: in the NIST corpus there is exactly one speaker per file, whereas in our broadcast news corpus, automatic speaker clustering is adopted, thus potentially generating some errors.

Not surprisingly, since the phonotactics module used the acoustic models of an ASR system trained for European Portuguese, the best performance of our language verification system was achieved for this language (2.5\% EER). A further analysis of the performance of the system revealed that the false alarms errors occurred mainly while misidentifying Galician speakers, and the missed detection errors appeared only on short files, some of them with much background noise or non-speech segments, erroneously classified as speech by the automatic audio preprocessing system. When tested over segments of duration above 10s, the equal error rate drops to $0.2 \%$ EER, and no errors were observed when considering segments above 20s. Hence we may consider that the language verification is robust enough to be integrated in our Broadcast News recognition system in order to exclude non-Portuguese speech segments, which was the real goal of this work.

A further experiment was conducted involving a different corpus which includes BN data from other varieties of Portuguese, namely the ones spoken in Brazil and in African countries with Portuguese as official language. In this experiment, the error rate is above the language verification error rate mentioned above $(10.6 \%$ for the 30 second test segments), but most errors seem again to come from the bad 
acoustic conditions of the test excerpts, which often contain loud background music (typically the jingles that mark headlines or weather forecast news).

This experiment showed that the verification system can cope with other varieties of Portuguese. However, some of these varieties can cause a severe degradation of the performance of the recognizer. Hence, the second part of this work was devoted to the study of an accent identification system for Portuguese, using this multi-variety BN corpus.

Our accent identification system using only 3 broad classes achieved an average correct identification rate of $83.9 \%$. The least confusable variety was by far BP ( $96.6 \%$ correct identification). EP was next. African varieties were the hardest to discriminate. When trying to discriminate between the African varieties themselves, the correct identification rate was only $42.0 \%$.

The results of these experiments were compared with the ones of a human benchmark test, which basically revealed a very good capacity for detecting BP and, although not so easily, EP, and similar difficulties in discriminating African varieties, although they could also be easily identified as such. The average 3-class identification ratio was $96.2 \%$ for sentences.

Finally, the results were also discussed in view of the performance of an EP-trained speech recognition system when confronted with other varieties. Given the strong degradation mainly for BP, the adaptation of the models of our EP-trained recognizer to these varieties is one of the topics we are currently pursuing.

There are many ways in which the above described language/variety identification methods can be improved. For instance:

- The PRLM can be improved by adding other languages phones to the phone recognizer, or by using several language-specific phone recognizers. Another point can be considering phone lattices, as proposed initially in [15] and used in the MIT system on the NIST 2005 language recognition data.

- The acoustic system can be improved by using different kinds of models: recent research has shown an interest in SVMs (especially for the language verification framework for which they are more suited). ANNs can also be investigated.

- The prosodic system can be modified using a better definition of a pseudosyllable, by taking into account the different types of vowels and consonants. An important issue is to take into account the variations in terms of speaking rate that can occur in different speaking styles.

- The fusion procedure can be much more sophisticated. For instance, one can implement a backend classifier using either Neural Networks or Fuzzy Logic algorithms. 


\section{Acknowledgments}

The authors would like to thank our colleagues Hugo Meinedo and Ernesto de Andrade for helpful comments. This work was partially funded by FCT under the post-doc scholarship SFRH/BPD/22032/2005, and also by the European project Vidi-Video, and by PRIME National Project TECNOVOZ number 03/165.

\section{References}

[1] M. B. Abaurre, E. G. Pagotto, Nasalização no Português do Brasil, in: I. Koch (ed.), Gramática do Português Falado, vol. VI, Editora da Unicamp/FAPESP, Campinas SP, 1996, pp. 495-526.

[2] R. André-Obrecht, A new statistical approach for automatic speech segmentation, IEEE Transactions on Acoustics, Speech and Signal Processing 36 (1) (1988) 29-40.

[3] P. Barbosa, E. Albano, Brazilian Portuguese - illustrations of the IPA, Journal of the International Phonetic Association 34 (2) (2004) 227-232.

[4] K. Berkling, M. Zissman, J. Vonwiller, C. Cleirigh, Improving accent identification through knowledge of english syllable structure, in: ICSLP'98, Sydney, Australia, 1998.

[5] D. Callou, Y. Leite, Iniciação à Fonética e Fonologia, Jorge Zahar Editor, Rio de Janeiro, 1990.

[6] W. Campbell, T. Gleason, J. Navratil, D. Reynolds, W. Shen, E. Singer, P. TorresCarrasquillo, Advanced language recognition using cepstra and phonotactics: MIT-LL system performance on the NIST 2005 LRE, in: Proceedings of Odyssey 2006: The Speaker and Language Recognition Workshop, 2006.

[7] E. Campione, J. Véronis, A multilingual prosodic database, in: International Conference on Spoken Language Processing, Sidney, 1998, http://www.lpl.univaix.fr/projects/multext.

[8] T. Chen, C. Huang, E. Chang, J. Wang, Automatic accent identification using gaussian mixture models, in: IEEE Workshop on Automatic Speech Recognition and Understanding (ASRU), 2001.

[9] R. M. Dauer, Stress-timing and syllable-timing reanalysed, Journal of Phonetics 11 (1983) 51-62.

[10] F. Fernandes, Subject localization constraints in BP and EP, Ph.D. thesis, Univ. Estadual Campinas (2007).

[11] S. Frota, M. Vigario, F. Martins, Language discrimination and rhythm classes: evidence from portuguese, in: Speech Prosody, Aix-en-Provence, France, 2002. 
[12] S. Frota, M. Vigário, On the correlates of rhythmic distinctions: The European / Brazilian Portuguese case, Probus 13.

[13] H. Fujisaki, Prosody, information and modeling - with emphasis on tonal features of speech, in: ISCA Workshop on Spoken Language Processing, Mumbai, India, 2003.

[14] P. Fung, L. Kat, Fast accent identification and accented speech recognition, in: ICASSP'1999, Phoenix, Arizona, 1999.

[15] J.-L. Gauvain, A. Messaoudi, H. Schwenk, Language recognition using phone lattices, in: INTERSPEECH'2004, Jeju, Korea, 2004.

[16] R. Huang, J. H. Hansen, Gaussian mixture selection and data selection for unsupervised spanish dialect classification, in: INTERSPEECH'2006, Pittsburgh, Pennsylvania, 2006.

[17] A. Ikeno, J. H. Hansen, The role of prosody in the perception of us native english accents, in: INTERSPEECH'2006, Pittsburgh, Pennsylvania, 2006.

[18] S. Kitazawa, Periodicity of Japanese accent in continuous speech, in: Speech Prosody, Aix en Provence, France, 2002.

[19] M. Komatsu, T. Arai, T. Sugawara, Perceptual discrimination of prosodic types, in: Speech Prosody, Nara, Japan, 2004.

[20] A. Lacerda, N. Rossi, Particularidades fonéticas do comportamento elocucional da fala do Rio de Janeiro (em confronto com o Português normal de Portugal), Revista do Laboratório de Fonética Experimental da Faculdade de Letras da Universidade de Coimbra IV (1958) 5-102.

[21] Y. Leite, D. Callou, J. Morais, Neutralização e realização fonética: a harmonia vocálica no Português do Brasil, in: Actas do Congresso Internacional sobre o Português, vol. III, Lisboa, 1996.

[22] J. Li, S. Yaman, C.-H. Lee, B. Ma, R. Tong, D. Zhu, H. Li, Language recognition based on score distribution feature vectors and discriminative classifier fusion, in: Proceedings of Odyssey06: The Speaker and Language Recognition Workshop, 2006.

[23] M. Lincoln, S. Cox, S. Ringland, A comparison of two unsupervised approaches to accent identification, in: ICSLP'98, Sydney, Australia, 1998.

[24] P. Matejka, L. Burget, P. Schwarz, J. Cernocký, Brno university of technology system for nist 2005 language recognition evaluation, in: Proceedings of Odyssey 2006: The Speaker and Language Recognition Workshop, 2006.

[25] M. H. Mateus, E. d'Andrade, The Phonology of Portuguese, Oxford University Press, Oxford, 2000.

[26] H. Meinedo, D. Caseiro, J. Neto, I. Trancoso, Audimus.media: a broadcast news speech recognition system for the european portuguese language, in: PROPOR'2003 6th International Workshop on Computational Processing of the Portuguese Language, 2003. 
[27] H. Meinedo, J. Neto, Audio segmentation, classification and clustering in a broadcast news task, in: ICASSP'2003, Hong Kong, 2003.

[28] H. Meinedo, J. Neto, A stream-based audio segmentation, classification and clustering pre-processing system for broadcast news using ANN models, in: INTERSPEECH'2005, 2005.

[29] S. Parkinson, Portuguese, in: M. H. . N. V. (eds.) (ed.), The Romance Languages, 1988, pp. 131-169.

[30] F. Pellegrino, R. André-Obrecht, Vocalic system modeling: A VQ approach, in: IEEE Digital Signal Processing, Santorini, 1997.

[31] J.-L. Rouas, Caractérisation et identification automatique des langues, Ph.D. thesis, Univ. Toulouse 3, France (2005).

[32] J.-L. Rouas, Modeling long and short-term prosody for language identification, in: INTERSPEECH'2005, Lisboa, Portugal, 2005.

[33] J.-L. Rouas, Automatic prosodic variations modelling for language and dialect discrimination, IEEE Transactions on Audio, Speech and Language Processing 15 (6) (2007) 1904-1911.

[34] J.-L. Rouas, M. Barkat-Defradas, F. Pellegrino, R. Hamdi, Identification automatique des parlers arabes par la prosodie, in: Journées d'Etude de la Parole, 2006.

[35] J.-L. Rouas, I. Trancoso, C. Viana, M. Abreu, Portuguese variety identificaiton on broadcast news, in: 2008 IEEE International Conference on Acoustics, Speech, and Signal Processing (ICASSP 2008), Las Vegas, Nevada, 2008.

[36] T. Schultz, Q. Jin, K. Laskowski, A. Tribble, A. Waibel, Speaker, accent, and language identification using multilingual phone strings, in: HLT'2002, San Diego, California, 2002.

[37] K. Sjölander, The snack sound toolkit.

URL http: //www. speech.kth.se/snack/

[38] A. Stolcke, Srilm - an extensible language modeling toolkit, in: INTERSPEECH'2002, Denver, Colorado, 2002.

URL http://www.speech.sri.com/projects/srilm/

[39] P. A. Torres-Carrasquillo, T. P. Gleason, D. A. Reynolds, Dialect identification using gaussian mixture models, in: Odyssey: The Speaker and Language Recognition Workshop, Toledo, Spain, 2004.

[40] W.-H. Tsai, W.-W. Chang, Discriminative training of gaussian mixture bigram models with application to Chinese dialect identification, Speech Communication 36 (3-4) (2002) 317-326.

[41] A. Vandecatseye, J.-P. Martens, J. Neto, H. Meinedo, C. Garcia-Mateo, J. Dieguez, F. Mihelic, J. Zibert, J. Nouza, P. David, M. Pleva, A. Cizmar, H. Papageorgiou, C. Alexandris, The COST 278 pan-european broadcast news database, in: LREC'2004, Lisbon, 2004. 
[42] B. Vieru-Dimulescu, P. B. de Mareüil, Perceptual identification and phonetic analysis of 6 foreign accents in french, in: INTERSPEECH'2006, Pittsburgh, USA, 2006.

[43] T. Wu, D. V. Compernolle, J. Duchateau, Q. Yang, J.-P. Martens, Improving the discrimination between native accents when recorded over different channels, in: INTERSPEECH'2005, Lisbon, Portugal, 2006.

[44] B. Yin, E. Ambikairajah, F. Chen, Combining cepstral and prosodic features in language identification, in: International Conference on Pattern Recognition, 2006.

[45] Y. Zheng, R. Sproat, I. Shafran, H. Zhou, Y. Su, D. Jurafsky, R. Starr, S. Yoon, Accent detection and speech recognition for shanghai-accented mandarin, in: INTERSPEECH'2005, Lisbon, Portugal, 2006.

[46] M. A. Zissman, Automatic language identification using gaussian mixture and hidden markov models, in: IEEE 18th International Conference on Acoustics, Speech, and Signal Processing, Minneapolis, MN, USA, 1993.

[47] M. A. Zissman, K. M. Berkling, Automatic language identification, Speech Communication 35 (1-2) (2001) 115-124. 